\title{
PROGRAMA EMOCIONAL EN ALUMNADO DE SEGUNDO DE E.S.O. CON DÉFICIT EN SUS LOGROS ACADÉMICOS
}

\author{
Lucía Morales-Sánchez \\ Laboratorio de Inteligencia Emocional. Universidad de Cádiz. \\ Puerto Real (Cádiz), España. \\ lucia.morales@uca.es \\ Paula Ruiz-González \\ Laboratorio de Inteligencia Emocional. Universidad de Cádiz. \\ Puerto Real (Cádiz), España. \\ Instituto de Investigación para el Desarrollo Social Sostenible INDESS. Universidad de Cádiz. Jerez de la \\ Frontera (Cádiz), España. \\ Ana Merchán-Clavellino \\ Laboratorio de Inteligencia Emocional. Universidad de Cádiz. \\ Puerto Real (Cádiz), España. \\ Instituto de Investigación para el Desarrollo Social Sostenible INDESS. Universidad de Cádiz. Jerez de la \\ Frontera (Cádiz), España. \\ Rocío Guil \\ Laboratorio de Inteligencia Emocional. Universidad de Cádiz. \\ Puerto Real (Cádiz), España. \\ Instituto de Investigación para el Desarrollo Social Sostenible INDESS. Universidad de Cádiz. Jerez de la \\ Frontera (Cádiz), España.
}

Fecha de Recepción: 18 Febrero 2018

Fecha de Admisión: 10 Abril 2018

\section{RESUMEN}

La gestión emocional se plantea como uno de los retos emergentes en la sociedad actual. En este sentido, el ámbito educativo se presenta como uno de los pioneros en el diseño e implementación de programas que promueven su desarrollo, favoreciendo en el alumnado sus competencias sociales y personales (Guil y Gil-Olarte, 2007).

De acuerdo con la normativa educativa vigente, las administraciones educativas promoverán el máximo desarrollo personal, intelectual, social y emocional del alumnado (Ley Orgánica 2/2006, de 3 de mayo, de Educación modificada por la Ley Orgánica 9/2013, de 9 de diciembre, para la Mejora de la Calidad Educativa). Sin embargo, en la Educación Secundaria Obligatoria (E.S.O.) encontramos escasos programas que atiendan a este desarrollo integral. Asimismo, este alumnado presenta necesidades específicas de apoyo educativo para alcanzar el logro académico debido, entre otras 
razones, a su déficit en diversas competencias emocionales y habilidades sociales (Santamaría y Valdés, 2017).

Concretamente, en Andalucía, el alumnado de Formación Profesional Básica presenta altas tasas de absentismo, así como escasa motivación, autoconcepto, autoestima y habilidades sociales, entre otras características (Junta de Andalucía, 2018). Para dar respuesta a esta situación, planteamos un proyecto piloto cuya finalidad será incrementar el logro académico y la adaptación socioescolar a través de las competencias emocionales. Dicho proyecto se ha llevado a cabo con cinco alumnos/as de segundo curso de E.S.O. que cumplen las características necesarias para iniciar las enseñanzas de Formación Profesional Básica en el próximo curso escolar. De este modo, se desarrollarán las competencias emocionales del alumnado con el refuerzo de las materias instrumentales y fundamentales del currículum de manera complementaria a la enseñanza formal.

Palabras clave: competencias emocionales; inteligencia emocional; rendimiento académico; formación profesional básica

\section{ABSTRACT}

Emotional trainning program in middle-school students with low academic performance.

Emotional management is considered one of the emerging challenges in current society. In this sense, the educational field appears as one of the pioneers designing and implementing of programs to promote its development, favoring in the students' social and personal skills (Guil and Gil-Olarte, 2007).

According to current educational laws in Spain, educational administrations should promote the maximum personal, intellectual, social, and emotional development of students (Organic Educational Law 2/2006, of May 3, modified by the Organic Law 9/2013, of 9 December, for the Improvement of Educational Quality). However, in Middle School (in Spain Secondary Mandatory School, ESO) we find few programs that address this integral development. Moreover, these students have special educational needs in order to achieve academic performance due, among others, to emotional competencies and social skills deficits (Santamaría and Valdés, 2017).

Specifically, in Andalusia, students of Basic Vocational Training, usually present high rates of absenteeism, as well as poor motivation, self-concept, self-esteem, and social skills (Junta de Andalucía, 2018). To respond to this situation, we propose a pilot project aimed to increase academic achievement and socio-academic adaptation through emotional competencies. This project has been developed with five students from eighth grade (second year of Spanish ESO) that met the basic criteria to begin Basic Vocational Training next academic year. In this way, students' emotional competencies will be developed through the reinforcement of instrumental and fundamental curricular subjects, complementary to formal education.

Keywords: emotional competences; emotional intelligence; academic performance; basic vocational training

\section{ANTECEDENTES:}

Las enseñanzas de Formación Profesional Básica, así como su implantación, ordenación y desarrollo se integran con el resto de enseñanzas de Formación Profesional Inicial del sistema educativo español. La creación de estas enseñanzas ha sido promovida con la puesta en marcha de la Ley Orgánica de Educación, de 3 de mayo de 2006, modificada por la Ley Orgánica 9/2013, de 9 de diciembre, para la Mejora de la Calidad Educativa (en adelante, LOE-LOMCE), sustituyendo de esta forma al antiguo Programa de Cualificación Profesional Inicial (PCPI). Se crea, por tanto, con el objetivo de evitar el abandono escolar temprano del alumnado, abrirle expectativas de formación y cualificación posterior y facilitar su acceso a la vida laboral (Orden de 9 de junio de 2015). Al finali- 
zar estos estudios el alumnado obtendrá el título Profesional Básico correspondiente, con valor académico y profesional, y se les permitirá el acceso a los Ciclos Formativos de Grado Medio. En el ámbito laboral dicho título tendrá los mismos efectos que el título de Educación Secundaria Obligatoria para el acceso a empleos públicos y privados (LOE-LOMCE).

El alumnado que opta por cursar estas enseñanzas suele cumplir una serie de características: experiencia continuada en fracaso escolar, baja autoestima y falta de confianza en sí mismo/a en los entornos de aprendizaje reglados, escasa motivación por el aprendizaje, riesgo de abandono del sistema educativo sin obtención de titulación, escasas habilidades de interacción social, lenguaje restringido y déficit en la simbolización, nivel bajo en hábitos y técnicas de estudio, así como bajas inquietudes formativas y laborales. Teniendo en cuenta dicho perfil, encontramos una serie de necesidades educativas en este alumnado que se traducen en aspectos fundamentales a trabajar con ellos: la mejora del autoconocimiento, autoestima y motivación; la proporción de herramientas y habilidades para la gestión de la carrera y la toma de decisiones académicas y vocacionales; la confianza en el cambio; la adquisición de hábitos y técnicas de estudio, así como compromisos y responsabilidades; mejora de los procesos cognitivos y metacognitivos básicos relacionados con el aprendizaje; y la enseñanza de habilidades de interacción social (Junta de Andalucía, 2018).

La adolescencia es una etapa en la que la inestabilidad emocional y el afrontamiento de crisis vitales ejercen un impacto en el desarrollo emocional del ser humano (Espín, 2013). No obstante, este colectivo es capaz de comprender y regular tanto sus emociones propias como ajenas (Pons et al., 2014). Sin embargo, si el adolescente carece de un adecuado asesoramiento que contribuya a su desarrollo emocional, sus emociones pueden volverse impulsivas y descontroladas (Bisquerra et al., 2012).

En este sentido, se ha demostrado cómo la regulación emocional juega un importante papel en el desarrollo social y académico de los jóvenes (Brackett, Lopes, Ivcevic, Mayer y Salovey, 2004; Mayer, Salovey y Caruso, 2004a, b; Salovey, Brackett y Mayer, 2004). La salud mental y el equilibrio psicológico de los estudiantes están influidos por su capacidad para atender a sus emociones, experimentar con claridad los sentimientos y reparar sus estados de ánimos negativos. Estos aspectos son, a su vez, determinantes en el rendimiento académico final del alumnado (Fernández-Berrocal, Extremera y Ramos, 2003; Gil-Olarte, Palomera y Brackett, 2006; Pérez y Castejón, 2007; Petrides, Frederickson y Furnham, 2004). Así, las personas con escasas habilidades emocionales son más proclives a experimentar estrés y dificultades emocionales durante sus estudios.

Además, se ha encontrado que la Inteligencia Emocional posee un efecto moderador entre las habilidades cognitivas y el rendimiento académico. Basado en ello, Guil y Gil-Olarte (2007) ponen de manifiesto la importancia de las habilidades socioemocionales sobre el rendimiento académico, además del efecto facilitador de la regulación emocional en el control de la atención y el desarrollo de la motivación intrínseca del alumnado.

Dado que no resulta complicado, la enseñanza obligatoria debe fomentar el aprendizaje social y emocional en las aulas, pues simultáneamente mejoraría la autorregulación y la habilidad para solucionar problemas en este colectivo (Guil y Gil-Olarte, 2007). Apoyándonos en la Teoría de las Inteligencias Múltiples (Gardner, 1983) la formación integral del alumnado debe combinar el entrenamiento de habilidades cognitivas y emocionales (Fonseca, 2007).

Siguiendo esta línea, y para dar respuesta a las necesidades del alumnado de segundo de E.S.0., -que cumple el perfil de posible alumno/a de Formación Profesional Básica para el siguiente curso y, que simultáneamente presenta problemas de desadaptación escolar-, hemos elaborado una propuesta relacionada con los programas de desarrollo socioemocional (Social and Emotional Learning, SEL). Se ha demostrado que la implementación de estos programas incrementa el éxito 
académico, mejora la relación entre profesorado y alumnado, y desciende conductas disruptivas de este último (Durlak, Weissberg, Dymnicki, Taylor y Schellinger, 2011; Mestre, Pérez, Guil, González y Núñez, 2017). Parece ser que la relación entre el entrenamiento de la Inteligencia Emocional y los beneficios socioemocionales que se extraen del desarrollo de competencias bajo el paraguas de los programas SEL suponen una buena inversión social y educativa (Belfield et al., 2015). No obstante, aún es criticada la viabilidad de la enseñanza de estas habilidades en contextos educativos, pues éstas se desarrollan a través de programas adyacentes y externos al currículum, y no de manera integrada y transversal dentro del plan de estudios (Brackett y Rivers, 2013; Nathason, Rivers, Flynn y Brackett, 2016).

Debido a la inexistencia de proyectos específicos para alumnado de E.S.O. que reúna el perfil mencionado con anterioridad y, al mismo tiempo, cubra sus necesidades educativas, elaboramos una propuesta desde el ámbito de la Orientación Educativa. Este programa será alternativo y complementario a la programación académica e incluirá tanto el refuerzo de materias curriculares como otras que, en nuestro trabajo, denominamos "materias no curriculares" (Educación Emocional, Técnicas para la mejora del estudio y Orientación académica y profesional). Este proyecto piloto integra el aprendizaje socioemocional en el currículo de este alumnado y aboga por la mejora de su rendimiento académico y, por ende, de su adaptación socioescolar.

\section{OBJETIVOS}

Presentar un proyecto piloto desarrollado con alumnado de segundo de E.S.O. en riesgo de abandono escolar, de promoción de competencias emocionales y de refuerzo de otras materias tanto curriculares como no curriculares.

Establecer la influencia entre el desarrollo de competencias emocionales sobre la adaptación socioescolar y el rendimiento académico del alumnado propuesto a través de la promoción de su autoestima, autoconcepto, motivación y sus habilidades sociales.

Justificar la necesidad e importancia de implantar programas de promoción de competencias emocionales en la E.S.O., y particularmente en las enseñanzas de Formación Profesional Básica.

\section{DESTINATARIOS}

Nuestro proyecto piloto se ha realizado con cinco alumnos/as de segundo curso de Educación Secundaria Obligatoria, con edades comprendidas entre 13 y 14 años, de entre los cuales tres eran chicas.

El criterio de inclusión fue encontrarse en riesgo de abandono escolar y, al mismo tiempo, haber sido propuesto por el Departamento de Orientación para cursar en el próximo año enseñanzas de Formación Profesional Básica.

\section{DESCRIPCIÓN DEL PROYECTO:}

Bajo el respaldo teórico planteado con anterioridad, desarrollamos un proyecto piloto que se llevó a cabo de manera paralela y, al mismo tiempo complementaria, a la enseñanza formal, realizándose de forma coordinada con el profesorado, el Departamento de Orientación y sus familiares. En el programa se han impartido tanto materias curriculares: Lengua Castellana y Literatura, Matemáticas e Inglés, como materias no curriculares: Educación Emocional, Técnicas para la mejora del estudio y Orientación Académica y Profesional.

El proyecto tuvo una duración de cinco semanas, y para desarrollarlo se crearon dos grupos, uno del alumnado de $2^{\circ}$ de ESO A, y otro de $2^{\circ}$ de ESO B, siendo los destinatarios un total de cinco alumnos/as.

Las materias curriculares (Lengua, Matemáticas e Inglés) se impartieron en el horario inicial 
establecido para cada alumno/a en su grupo de origen. No obstante, para el desarrollo de las materias no curriculares (Educación Emocional, Orientación Académica y Profesional y Técnicas para la mejora del estudio), se fusionaban ambos grupos.

En total, se desarrollaron a la semana quince sesiones de una hora, fraccionadas en: dos sesiones grupales de Educación Emocional y una sesión dividida en el desarrollo de la Orientación Académica y Profesional y la impartición de técnicas para la mejora del estudio y, para cada grupo, dos sesiones de lengua, dos sesiones de matemáticas, y dos sesiones de inglés.

Aunque el programa lo constituyeron un total de seis materias (tanto curriculares como no curriculares), el eje clave y el aspecto innovador de este programa fue introducir el desarrollo de competencias emocionales como materia independiente dentro del mismo. No obstante, se trató de poner en práctica los contenidos trabajados en la asignatura de "Educación Emocional" en las demás materias. Todo ello con la finalidad de desarrollar, de manera simultánea a las competencias propias de cada asignatura, competencias emocionales relativas a la autonomía emocional, la competencia social y las habilidades de vida y bienestar que considera Bisquerra (2012). Se trabajó, fundamentalmente, la motivación, las habilidades sociales, la expresión de emociones, la tolerancia a la frustración, la identificación de problemas y la resolución de conflictos en este alumnado. $1)$ :

La organización de materias de nuestro proyecto queda reflejada en la siguiente tabla (Ver tabla

Tabla 1. Materias del proyecto

\begin{tabular}{ll}
\hline Materias curriculares & Materias no curriculares \\
\hline Lengua castellana y literatura & Educación emocional \\
Matemáticas & Hábitos y técnicas de estudio \\
Inglés & Orientación académica y profesional \\
\hline
\end{tabular}

Centrándonos en la materia de Educación Emocional, se atendió fundamentalmente a la función adaptativa de las emociones, específicamente, al conocimiento e identificación de las emociones propias y de los demás, así como a la expresión de las mismas. Todo ello se realizó a través de actividades y dinámicas de grupo que promovían habilidades sociales y comunicativas (empatía, asertividad, expresión oral, escucha activa y respeto), el autoconcepto, la autoestima y la motivación a través de la cohesión y el trabajo grupal.

En las diez sesiones de educación emocional se trabajaron los siguientes contenidos (ver Tabla 2): 
Tabla 2. Contenidos de las sesiones de Educación Emocional

\begin{tabular}{ll}
\hline Sesión 1 & Presentación de la materia. \\
& La Educación emocional. \\
& Autoconcepto y autoestima. \\
\hline Sesión 2 & Claridad emocional. \\
\hline Sesión 3 & Emociones básicas y sociales. \\
\hline Sesión 4 & Autoconocimiento. \\
\hline Sesión 5 & Autoconcepto. \\
\hline Sesión 6 & Autoestima. \\
\hline Sesión 7 & Regulación emocional. \\
\hline Sesión 8 & Conocimiento y expresión de emociones \\
\hline Sesión 9 & Empatía. \\
\hline Sesión 10 & Autoconocimiento, Autoestima, Empatía y Habilidades Sociales. \\
\hline
\end{tabular}

Teniendo en cuenta que nuestro trabajo se basa en el modelo de orientación por programas, nuestras acciones atendieron a los ejes de intervención directa, grupal, interna, así como reactiva: Es grupal, ya que se dirigió a todo el grupo de alumnos/as. Las actividades se desarrollaron de forma colectiva, aunque en casos excepcionales en los que la ocasión lo requería, se llevaron a cabo prácticas para trabajar individualmente; directa, porque la orientadora se encontró cara a cara con los destinatarios/as del proyecto; interna debido a que la intervención se produjo en el propio centro y por parte de profesionales del mismo; así como reactiva, ya que trató de responder a la necesidad de trabajar con este alumnado las competencias socioemocionales, así como el refuerzo de otras materias tanto curriculares como no curriculares.

En cuanto a la metodología didáctica utilizada en esta materia no curricular, se desarrollaron sesiones dinámicas, participativas, activas, reflexivas, individuales y grupales, así como dialógicas. En cada sesión se trabajaron los contenidos correspondientes, reflejados en la tabla anterior. Se propició el trabajo en grupo, creando actividades donde se pudieran abrir espacios de debate, se promoviese el diálogo y habilidades sociales y comunicativas. La importancia de utilizar técnicas grupales reside, entre otros motivos, en que permiten el entrenamiento personal mediante el ensayo de modos adecuados de resolver conflictos y/o gestión adecuada de las emociones, desarrollo de la empatía, etc. a través del aprovechamiento de los eventos o conflictos que surgen a diario en el propio grupo (Guil y Gil-Olarte, 2007).

\section{HALLAZGOS ENCONTRADOS}

Para conocer la efectividad del programa se recurrió a técnicas cualitativas: Observación Participante, Diario de Campo y análisis de información a partir de cuestionarios cualitativos respondidos por el alumnado, sus familiares y sus tutores/as.

Las respuestas en los cuestionarios del alumnado ponen de manifiesto su percepción sobre la utilidad de la Educación Emocional en la vida diaria, así como su influencia sobre su autoestima, seguridad y confianza, bienestar subjetivo y relaciones sociales. A su vez, han indicado cómo haber 
trabajado la Educación Emocional a lo largo del proyecto, así como el tratamiento alternativo de las materias curriculares del mismo, repercutió positivamente sobre su atención e interés en estas materias, fundamentalmente en matemáticas e inglés.

Por otro lado, el profesorado destacó la relevancia de promover la Educación Emocional para impulsar el desarrollo personal, académico y profesional futuro del alumnado. Asimismo, indicaron fundamental la promoción de la motivación, pues observaron que ésta repercutió positivamente en el desarrollo del alumno/a en diversos niveles, incluidos en el progreso de las demás asignaturas con el grupo-aula.

De la información extraída de las familias se pone de manifiesto que nuestro proyecto ha favorecido al alumnado en sus ámbitos académico y familiar, observándose en éstos: un aumento de su motivación en general y de su interés por realizar tareas, reducción de conductas disruptivas tanto en el centro como en el entorno familiar, así como un mayor interés por asistir a clases y por su futuro profesional.

Por último, es necesario considerar que la asistencia del alumnado al proyecto ha sido de un $90 \%$, por lo que se ha logrado motivar al mismo para cumplir los objetivos del curso en las diferentes materias curriculares. Se ha producido, además, una mejora en la adaptación del centro y mejores resultados académicos. Con la participación de los/as alumnos/as en el programa hemos prevenido el abandono escolar temprano, ya que éste era uno de los principales factores de riesgo de nuestra muestra.

\section{CONCLUSIONES}

Tras la aplicación de nuestro proyecto, los resultados parecen confirmar la necesidad latente que existe de desarrollar programas que promuevan las competencias emocionales en el alumnado de secundaria, sobre todo en aquél que presenta un perfil de riesgo (conductas disruptivas, escasa motivación e interés en el aula, baja autoestima y una valoración negativa de sí mismo/a). Basándonos en la información aportada por el profesorado del centro, se podría concluir que estos programas promueven simultáneamente el rendimiento académico y previenen la desadaptación socioescolar del alumnado, pues se observó una reducción del absentismo escolar y mayor participación en el aula.

Apoyándonos en la revisión de la literatura y los resultados obtenidos, consideramos esencial implementar programas que potencien aspectos clave de la Educación Emocional como el autoconcepto, la autoestima, las habilidades sociales y la automotivación en el alumnado que presenta riesgo de abandono escolar temprano.

Confirmamos, del mismo modo, la importancia de promover el desarrollo de estas competencias de manera transversal al currículum. Pues se ha demostrado que toda actividad cognitiva escolar unida al desarrollo de las capacidades emocionales y sociales también fomenta, ya sea de manera directa 0 indirecta, el desarrollo de la Inteligencia Emocional. Este aspecto es crucial en la evidencia de la conexión entre IE y rendimiento académico (Mestre, Guil y Gil-Olarte, 2004). Para el óptimo desarrollo de este tipo de programas es especialmente importante la formación del profesorado en competencias emocionales.

Como limitación principal de nuestra propuesta, se encuentra el no haber podido acceder a las calificaciones finales de los/as alumnos/as. Sin embargo, mediante y tras la realización de nuestro proyecto se contempló un progreso en las materias curriculares a lo largo de su aplicación. Por tanto, a partir de análisis de información cualitativa observamos una estrecha relación entre el desarrollo emocional del alumnado e indicadores de rendimiento académico.

Por último, proponemos como línea de investigación futura ampliar el tamaño muestral e incluir 
análisis cuantitativos de los resultados, como análisis estadísticos de correlación y regresión, que establezcan la relación entre la Inteligencia emocional y el rendimiento académico en alumnado en riesgo de abandono escolar. En especial, aquél que presenta el perfil comentado con anterioridad y que se pretende proponer para cursar las enseñanzas de Formación Profesional Básica en cursos posteriores.

\section{REFERENCIAS}

Belfield, C., Bowden, B., Klapp, A., Levin, H., Shand, R. y Zander, S. (2015). The economic value of social and emotional learning. New York, Estados Unidos: Center for Cost Benefit Studies in Education.

Bisquerra, R. (2012). Orientación, tutoría y educación emocional. Madrid, España: SÍnTESIS.

Bisquerra, R., Punset, E., Mora, F., García-Navarro, E., López-Cassá, E., Pérez-González, J. C. y Panells, 0. (2012). ¿Cómo educar las emociones? La inteligencia emocional en la infancia y la adolescencia. Esplugues de Llobregat, Barcelona: Hospital Sant Joan de Déu.

Brackett, M. A., Lopes, P. N., Ivcevic, Z., Mayer, J. D., y Salovey, P. (2004). Integrating emotion and cognition: The role of emotional intelligence. In D. Dai y R. J. Sternberg (Eds.), Motivation, emotion, and cognition: Integrating perspectives on intellectual functioning (pp. 175-194). Mahwah, Estados Unidos: Lawrence Erlbaum.

Brackett, M. A. y Rivers, S. E. (2013). Transforming Students' Lives with Social and Emotional Learning. En R. Pekrun y L. Linnenbrink-Garcia (eds.), International Handbook of Emotions in Education (pp. 368-388). New York, Estados Unidos: Taylor and Francis.

Downey, L., Johnston, P., Hansen, K., Birney, J. y Stough, C (2010). Investigating the mediating effects of emotional intelligence and coping on problem behaviours in adolescents, Australian Journal of Psychology, 62, pp. 20-29. doi: 10.1080/00049530903312873

Durlak, J. A., Weissberg, R. P., Dymnicki, A. B., Taylor, R. D. y Schellinger, K. B. (2011). The impact of enhancing students' social and emotional learning: A metaanalysis of school-based universal interventions. Child Development, 82, 405- 432. doi:10.1111/j.1467-8624.2010.01564.x.

Espín, M. C. (2013). Crecer en la adolescencia. Revista Sexología y Sociedad, 2(5), 2-6.

Fernández-Berrocal, P., Extremera, N. y Ramos, N. (2003a). Inteligencia emocional y depresión. Encuentros en Psicología Social, 1(5), 251-254.

Fonseca-Mora, M.C. (2007). Las inteligencias múltiples en la enseñanza del español: Los estilos cognitivos de aprendizaje. En Pastor Villalba, C. (ed.). Actas del Programa de Formación para profesorado de español como lengua extranjera. Múnich, Alemania: Instituto Cervantes.

Gardner, H. (1983). Frames of Mind: the Theory of Multiple Intelligences. Nueva York, Estados Unidos: Basic Books.

Gil-Olarte, P., Palomera, R. y Brackett, M.A. (2006). Relating emotional intelligence to social competence and academic achievement among high school students, Psicothema, 18, 118-123.

Guil, R. y Gil-Olarte, P. (2007). Inteligencia emocional y Educación: Desarrollo de competencias socio-emocionales en Mestre, J.M. y Fernández, P. (coords). Manual de Inteligencia Emocional. Madrid, España: Pirámide.

Junta de Andalucía (2018). La tutoría en la F.P. Básica. Recursos y Materiales. Planificación. Consejería de Educación: Junta de Andalucía. Recuperado de: http://www.juntadeandalucia.es/educacion/webportal/web/tutoria-fp-basica/planificacionaccion-tutorial

Ley Orgánica 2/2006, de 3 de mayo, de Educación modificada por la Ley Orgánica 9/2013, de 9 de diciembre, para la Mejora de la Calidad Educativa. Boletín Oficial del Estado, núm. 295, de 10 de 
diciembre de 2013, pp. 97858 a 97921. http://www.boe.es/boe/dias/2013/12/10/pdfs/BOE-A2013-12886.pdf.

Mayer, J.D., Salovey, P., y Caruso, D.R. (2004a). Emotional intelligence: theory, findings and implications, Psychological Inquiry, 60, 197-215.

Mayer, J.D., Salovey, P., y Caruso, D.R. (2004b). A further consideration of issues of emotional intelligence, Psychological Inquiry, 60, 249-255.

Mestre, J. M., Guil, R. y Gil-Olarte, P. (2004). Inteligencia Emocional: algunas respuestas empíricas y su papel en la adaptación escolar en una muestra de alumnos de secundaria, Revista Electrónica de Motivación y Emoción, VII, 6, Recuperado de: http://reme.uji.es/articulos/numero22/article5/numero\%2022\%20article\%20 5\%20INTEL.pdf

Mestre, J.M., Pérez, N., Guil, R. González, G., Núñez, J.M. (2017). El desarrollo de la Inteligencia Emocional a través de la optimización de las capacidades cognitivas a través de la Educación Obligatoria, Contextos educativos, 20, 57-75. Doi: http://doi.org/10.18172/con.3023

Mestre, J. M., Guil, R., Lopes, P., Salovey, P. y Gil-Olarte, P. (2006). Emotional intelligence and social and academic adaptation to school, Psicothema, 18, 112-118.

Morales, F.M. (2017). Relaciones entre afrontamiento del estrés cotidiano, autoconcepto, habilidades sociales e inteligencia emocional, European Journal of Education and Psychology, 10, 4148.

Nathason, L., Rivers, S., Flynn, L. y Brackett, M. (2016). Creating Emotional Intelligence Schools with RULER, Emotion Review, 8(4), 305-310. DOI: http://doi. org/10.1177/1754073916650495.

Orden de 9 de junio de 2015, por la que se regula la ordenación de las enseñanzas de Formación Profesional Básica en Andalucía para los cursos académicos 2014/2015 y 2015/2016, se establece el procedimiento de escolarización para el curso académico 2015/2016 y se desarrollan los currículos correspondientes a veinte títulos profesionales básicos. Boletín oficial de la Junta de Andalucía, núm. 124, de 29 de junio de 2015, pp. 1-1155. http://www.juntadeandalucia.es/boja/2015/124/B0JA15-124-01155.pdf.

Pérez, N. y Castejón, J. (2007). La Inteligencia emocional como predictor del rendimiento académico en estudiantes universitarios, Ansiedad y estrés, 13 (1), 119-129.

Petrides, K. V., Frederickson, N. y Furnham, A. (2004). The role of trait emotional intelligence in academic performance and deviant behavior at school, Personality and Individual Differences, 36 (2), 277-293.

Pons, Francisco; de Rosnay, M.; Bender, P.; Doudin, Pierre-André; Harris, P. y Giménez-Dasí, M. (2014). The impact of abuse and learning difficulties on emotion understanding in late childhood and early adolescence, The Journal of Genetic Psychology: Research and Theory on Human Development, 175(4), 301-317. Doi: 10.1080/00221325.2014.903224.

Salovey, P., Brackett, M.A., y Mayer, J.D. (2004). Emotional intelligence: key readings on the Mayer and Salovey model. Port Chester, Estados Unidos: Dude Press.

Santamaría, B. y Valdés, M.V. (2017). Rendimiento del alumnado de Educación Secundaria Obligatoria: influencia de las habilidades sociales y la inteligencia emocional. International Journal of Developmental and Educational Psychology. INFAD Revista de Psicología, no1 Monográfico 1, pp. 57-66. ISSN: 0214-9877.

Wang, Y., Xie, G. y Cui, X. (2016). Effects of emotional intelligence and self-leadership on students' coping with stress, Social Behavior and Personality, 44, pp. 853-864. Doi: 10.2224/sbp.2016.44.5.853. 
